\title{
Comparative Analysis of Image Denoising Algorithms using Reference Based Image Quality Measures
}

\author{
Iqra Saleem \\ Department of Computer Systems Engineering, \\ University of Engineering and Technology, Peshawar, Pakistan \\ Email: saleemiqra295@gmail.com
}

\begin{abstract}
Much research has been dedicated to find the best denoising method for the specific noise model present in an image. Various linear filters, such as mean filter, Arithmetic mean, Contra harmonic and Weiner filter are becoming increasingly common in removing Gaussian and salt and pepper noise in images. Nonlinear filters like median and Alpha Trimmed filters play avital role in eliminating impulsive noise. This paper demonstrate a comparative analysis of various popular noise suppression approaches to find the appropriate and consistent technique for denoising images. The experimental outcomes are discussed and analysed to find the overall benefits and drawbacks of each technique.
\end{abstract}

Key words: Gaussian noise, Image Denoising Technique, Salt-and-pepper noise, Speckle noise.

\section{INTRODUCTION}

Digital Images contain significant information in the object or in the form of text which can be utilized in many practical applications such as communications, astronomy and geography. These images may contain degradations due to various reasons. Blurring can occur in images due to imperfect image capturing process such as relative motion between camera and original scene. Similarly, in aerial photographs blurs are introduced by atmospheric turbulence or relative motion between sensor and ground. Besides this, noise can also be introduced in images when we want to store them as digital. This process is called quantization. Noisy channels, electronic and photometric disorders, transmission and compression are also the main causes of noise.

Thus noise removal is often essential and pre-processing step to improve and recover minute details in image before using in other applications. There are many practical applications of image denoising algorithms such as in object and face detection, text recognition, in the field of photography and medicine and forensic investigation.
Image denoising is still a challenging task for researchers because there is always a trade-off between removing noise and preserving edges and other fine details in image. This paper explains various noise reduction techniques and also gives us an idea about which algorithm works best by using quality measure techniques between original and degraded image. Various noise models have been discussed on the basis of error source. All type of noises are also discussed in this paper in detail. Different denoising algorithms are used for specific noise model. It means an algorithm that is used to remove noise from aerial photographs may not be suitable for medical images.

The scope of this paper is to analyse different noise models and their suppression techniques. We have included experimental results and comparison of different filters. This paper is organized as follow. In section 2 related work is discussed. In section 3 various noise models are defined. Section 4 explain different denoising algorithms. Section 5 gives the discussion about reference based quality measures. Section 6 is about the experimental results. Finally, conclusion is presented in section 7.

\section{RELATED WORK}

A lot of work has been done on various denoising algorithms. Ruchika Shukla [1] demonstrated various image processing and restoration techniques to improve the overall quality of images. Authors also present different type of noises and their reduction techniques. P.Patidar el.al [2] presented brief overview of various denoising techniques. In this paper, authors present a complete and quantitative analysis of various noise models.

Suhaila Sari et.al [3] develop a way for noise reduction by using different type of edge preserving filters like bilateral filters and wavelet thresholding. The main drawback of this technique was that it is not appropriate for impulsive type of noise like salt and pepper noise. Improved Non-Local Means Algorithm was proposed by Lingli Huang et.al [4]. This technique computes weighted pixels from the adjacent pixels and replace all pixels with them. Experimental outcomes show that the process increases the denoising performance. I. M. El- 
Henawy [5] presented various techniques for image deblurring. Blurring can occur in images due to various reasons like relative motion between object and capturing device and low light.

A. Jaiswal et.al [6] presented a method for denoising Gaussian and salt and pepper noise both at a time. In the first step noise is reduced in image by filtering. Then apply wavelet based filtering techniques on the same image. In second step, thresholding and denoising is applied on image simultaneously. By using image quality measure, PSNR, results are calculated for all cases. Noises and Image Denoising Techniques [7] review the existing noise reduction algorithms, such as filtering and wavelet based techniques, multifractal approach, and performs their comparison. This algorithm works best for salt and pepper noise. The wavelet based approach works best when the image is degraded with Gaussian noise. Median filter works well for removing noise especially salt and pepper noise while having edge preserving properties [8].

Weighted median filter is suitable for denoising salt and pepper noise in grayscale images as compared to standard and adaptive filters. This method process only noisy pixels in image [9]. Gabriela Ghimpe, teanu et.al [10] demonstrated a model that finds the image's components in moving frame. This approach was developed to denoise the components in moving frame as well as preserving geometry.

\section{NOISE MODELS}

Noise is an unwanted disturbance that degrades the quality of an image. It is random variation in image pixels. Noise can be add to image from different sources such as from noisy channel, electronic transmission, faulty instruments and compression [11]. Original image of Leema is shown in Figure 1.

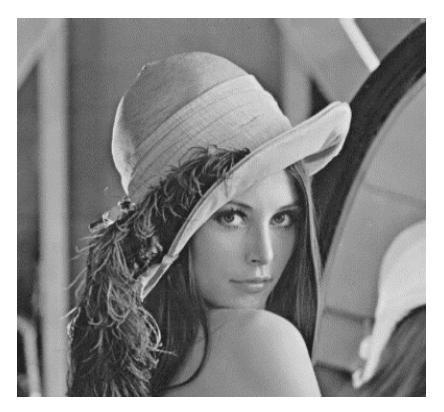

Figure 1: Original Image of Lena [12]

Noise in image is either multiplicative or in additive form.

\subsection{Additive Noise}

When noisy signal added to the original signal and produce corrupted image is known as additive noise such as additive Gaussian noise.

$$
W(i, j)=s(i, j)+n(i, j)
$$

\subsection{Multiplicative Noise}

When noisy signal multiplied to the original signal and produce corrupted image is known as multiplicative noise such as speckle noise.

$$
W(i, j)=s(i, j) \times n(i, j)
$$

$s(i, j)$ denotes the original image intensity, $n(i, j)$ represents noise ad $\mathrm{W}(\mathrm{i}, \mathrm{j})$ is corrupted image. There are various ranges of noises having different characteristics as follow:

\section{a. Salt and Pepper Noise}

This is an impulsive noise and also known as spike noise. It seems like scattering of both bright and dim spots in image. Image with this type of noise contain dark pixels in the bright regions and bright pixels in the dark regions [13].

For an 8- bit image, the value for pepper noise is less i.e. 0 and for salt noise is high i.e. 1 . Hence the distorted image contains only two values, either extreme low or extreme high value. This type of noise is generally occur due to faulty camera sensors, sudden disturbance, during transmission or through errors caused by analog-to-digital converters. This spike noise can be eliminate by using filtering technique and by using dark frame subtraction. Salt and pepper noise is shown in Figure. 2

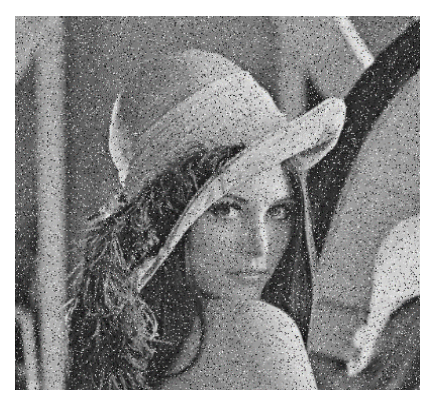

Figure 2: Lena Image with Salt and Pepper noise

\section{b. Gaussian Noise}

Also called as additive noise. It is evenly distributed over every pixel in an image. The corrupted image's pixels is due to addition of true pixel value and random Gaussian noise value [14]. At every point, intensity of pixel value is different from every other pixel. Gaussian noise arise in images is due to low illumination during image capturing, difference in temperature and electronic and sensor noise. Various spatial filters like median, mean filters are used for smoothing the image but it introduce blurs and fails to preserve edges. Gaussian noise is 
shown in Figure 3. The probability density function (PDF) is shown in the following equation:

$$
p_{G}(z)=\frac{1}{\sigma \sqrt{2 \pi}} e^{-\frac{(z-\mu)^{2}}{2 \sigma^{2}}}
$$

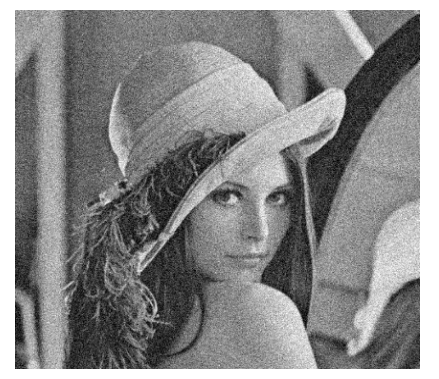

Figure 3: Lena Image with Gaussian noise

\section{c. Speckle Noise}

This is multiplicative noise. It causes distortion by multiplying each pixel with noise value in an image. Medical images like ultrasound images contain speckle noise. It is mostly observed in laser images. This distortion greatly affect Synthetic aperture radar (SAR) images [15].This type of noise arises due to accidental fluctuations in the incoming signal from. It increases the mean grey level of corrupted image. Presence of this noise causes problems in image interpretation. Image with speckle noise is shown in Figure 4.

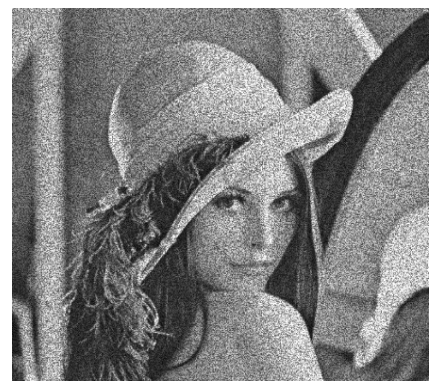

Figure 4: Lena Image with Speckle noise

\section{IMAGE FILTERING TECHNIQUES}

Image denoising is very important task for increasing the image quality before using them in other practical applications. There are various filtering techniques each having its own pros and cons. The main goal of filtering is removing noise as well as preserving the edges. One can choose the filtering algorithm according to the amount of noise present in an image and type of noise.

There are two approaches for reducing distortions in images: Linear Filtering and Non-linear Filtering. In linear filtering, denoising algorithm is implemented linearly on all noisy and non-noisy pixels. Non-linear filters are used on pixels specifically adjacent with noisy pixels. The two filters are categorized as follow:

Linear Filters:
a) Arithmetic Mean
b) Weiner Filter
c) Harmonic
d) Contra harmonic

Non-Linear Filters:
a) Max and Min
b) Alpha Trimmed
c) Media

\section{a. Mean Filter}

Mean filter is a basic smoothing filter that replaces the mid value of the window with the average values of its all adjacent pixels values [16]. It decreases the intensity variations between the neighbouring pixels. It is applied with kernel mask, which provides the weighted sum of values of a pixel and its neighbours. This filter is easy to implement but the main limitation of mean filter is that edges get blurred while smooth out the image. The equation for mean filter is shown below where $G(i, j)$ is the sub image area at $(i, j)$ and $g(x, y)$ is the sub image area of $m * n$.

$$
G(i, j)=\frac{1}{m} \sum(x, y) \in s_{i j} g(x, y)
$$

\section{b. Median Filter}

In median filter, there is also a sliding window concept like mean filter. The kernel slides over the pixels values in image and find the median value which is replaced by the centre pixel value of the window. It first rearrange all the adjacent pixel values into numerical order and then replace the mid value. Median filter is good in image smoothing as well as in preserving sharp edges and fine details. Figure 5. Shows the median calculation.

\begin{tabular}{|l|l|l|l|l|}
\hline 124 & 123 & 130 & 140 & 150 \\
\hline 111 & 120 & 150 & 125 & 135 \\
\hline 119 & 115 & 119 & 123 & 134 \\
\hline 118 & 124 & 126 & 127 & 133 \\
\hline 122 & 122 & 129 & 131 & 137 \\
\hline
\end{tabular}

Adjacent Values: $115,119,120,123,124$, $125,126,127,150$

Figure 5: Median Filtering

Median Value is: 124

\section{c. Weiner Filter}

This filter is based on statistical approach and is used to denoising images. For this filter, prior knowledge of linear time-variant and the properties of original signal and the noise is important like spectral properties etc. Weiner filter is a kind of adaptive filter which is more appropriate for preserving edges and other high frequency details in image

\section{d. Adaptive Median Filter}

This adaptive filter works very well on the images having salt and pepper noise. It smooths the image as well as preserve 
sharp edges and fine details. Its kernel size varies according to different situations. This filter first computes median value of degraded image and finds whether the resulted median value belongs to salt or pepper noise or other additive noise. If it is median of impulsive noise then it increases the size of window and recomputes all the above values again otherwise it jumps to second stage in which it checks whether the nominated pixel is a salt or pepper noise or not. If it is, then it replaces the nominated pixel with median value which is computed earlier otherwise the pixel remains the same.

\section{e. Arithmetic Mean Filter}

Just like mean filter, it also replaces the mid value by taking the average of surrounded pixels [17]. This type of filter is assumed to remove Gaussian noise. Advantage of this filter is that it smooths the image but increase blurring.

\section{f. Contra Harmonic Mean Filter}

This is a non-linear filter used for removing salt and pepper noise but works well for Gaussian noise. It is very good in preserving edge as compared to arithmetic mean filter. This filter performs well in eliminating positive and negative outlier for different cases. The equation for contra harmonic is shown in the below (5). For positive values of Q, it decreases pepper noise and for negative values of $\mathrm{Q}$, it decreases salt noise. It cannot perform both the function at the same time.

$$
f(x, y)=\frac{\sum_{(s, t) \in S_{x y}} g(s, t)^{Q+1}}{\sum_{(s, t) \in S_{x y}} g(s, t)^{Q}}
$$

\section{REFERENCE BASED PERFORMANCE PARAMETERS}

There are many reference based quality measures available in which quality of distorted image is measured in the presence of original image.

Mean Square Error: It is the mean squared difference between original and distorted image as shown below in equation 6, where $\mathrm{X}$ represents original image and $\mathrm{Y}$ represents noise image. $\mathrm{M}$ and $\mathrm{N}$ represents numbers of columns and rows and $i$ and $j$ represent the index of rows and columns respectively.

$$
M S E=\frac{1}{N M} \sum_{i=0}^{N-1} \sum_{j=0}^{M-1}[X(i, j)-Y(i, j)]^{2}
$$

Peak Signal to Noise Ratio: This quality measure show the ratio between maximum values of noise image with respect to that of original image. The higher the PSNR value, the better will be quality of filtered image. PSNR is shown in expression below (7) where $\mathrm{MAX}_{1}$ is the highest value that is present in our original image and MSE is mean square error.

$$
P S N R=10 \log _{10}\left(\frac{M A X_{i}^{2}}{M S E}\right)
$$

\section{EXPERIMENTAL RESULTS}

In the present study, we perform experiments on the original lena.bmp image. First we add different noises like impulsive noise, additive Gaussian and speckle noise on images and then apply different denoising algorithms on different noise models. Filters used in this paper are: Mean filter, Median Filter, Contra Harmonic Filter, Arithmetic Median Filter and Weiner Filter. Then MSE and PSNR quality measures are used to check the performance of each filter with respect to different noise models.

Below Table 1. Shows the PSNR values of distorted image with respect to different noise filters.

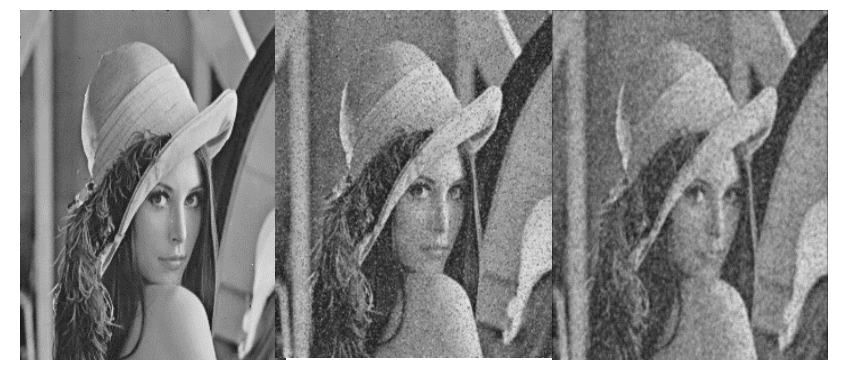

Figure 1:(a) Salt and Pepper Figure 1 (b) Salt and Pepper Figure 1 (c) Salt and Pepper Removal with adaptive Removal with Arithmetic Removal with Contra Median filter Mean Filter Harmonic Mean Filter

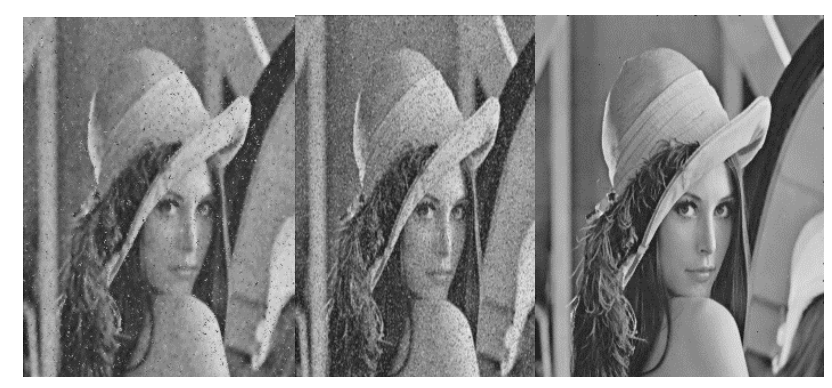

Figure 1 (d): Salt and Pepper Figure.1 (e) \{Salt and Pepper Figure1 (f) \{ Salt and Pepper Noise Removal with Weiner removal with Average removal with median Filter.

$$
\text { Filter. } \quad \text { Filter. }
$$




\begin{tabular}{|l|l|l|l|l|l|l|}
\hline \multicolumn{7}{|c|}{ Table 1. Salt and Pepper noise Filtering } \\
\hline $\begin{array}{l}\text { Salt and } \\
\text { Pepper } \\
\text { Noise }\end{array}$ & $\begin{array}{l}\text { Average } \\
\text { Filter }\end{array}$ & $\begin{array}{l}\text { Median } \\
\text { Filter }\end{array}$ & $\begin{array}{l}\text { Adaptive } \\
\text { Median } \\
\text { Filter }\end{array}$ & $\begin{array}{l}\text { Arithmetic } \\
\text { Mean filter }\end{array}$ & $\begin{array}{l}\text { Contra } \\
\text { harmonic } \\
\text { Mean Filter }\end{array}$ & $\begin{array}{l}\text { Weiner } \\
\text { Filter }\end{array}$ \\
\hline $\begin{array}{l}\text { In } \\
\text { percentage) }\end{array}$ & $P S N R$ & $P S N R$ & PSNR & PSNR & PSNR & PSNR \\
\hline $\mathbf{1 0} \%$ & 23.85 & 33.10 & 36.17 & 25.16 & 24.03 & 23.26 \\
\hline $\mathbf{2 0} \%$ & 20.79 & 28.70 & 29.94 & 22.73 & 20.86 & 21.42 \\
\hline $\mathbf{3 0} \%$ & 18.82 & 23.59 & 23.99 & 20.85 & 18.84 & 20.06 \\
\hline $\mathbf{4 0 \%}$ & 17.34 & 18.89 & 19.03 & 19.34 & 17.35 & 18.96 \\
\hline $\mathbf{5 0} \%$ & 16.13 & 15.30 & 15.36 & 18.03 & 16.12 & 17.91 \\
\hline
\end{tabular}

\begin{tabular}{|l|l|l|l|l|l|l|}
\hline \multicolumn{7}{|c|}{ Table 2. Gaussian Noise Filtering } \\
\hline $\begin{array}{l}\text { Gaussian } \\
\text { Noise }\end{array}$ & $\begin{array}{l}\text { Average } \\
\text { Filter }\end{array}$ & $\begin{array}{l}\text { Median } \\
\text { Filter }\end{array}$ & $\begin{array}{l}\text { Adaptive } \\
\text { Median } \\
\text { Filter }\end{array}$ & $\begin{array}{l}\text { Arithmetic } \\
\text { Mean filter }\end{array}$ & $\begin{array}{l}\text { Contra } \\
\text { harmonic } \\
\text { Mean Filter }\end{array}$ & $\begin{array}{l}\text { Weiner } \\
\text { Filter }\end{array}$ \\
\hline $\begin{array}{l}\text { In } \\
\text { percentage })\end{array}$ & PSNR & PSNR & PSNR & PSNR & PSNR & PSNR \\
\hline $\mathbf{0 . 0 1}$ & 27.64 & 26.75 & 23.05 & 27.30 & 28.18 & 28.50 \\
\hline $\mathbf{0 . 0 2}$ & 25.57 & 24.16 & 20.28 & 26.44 & 25.87 & 26.87 \\
\hline $\mathbf{0 . 0 3}$ & 24.26 & 22.60 & 18.70 & 25.76 & 24.47 & 25.76 \\
\hline $\mathbf{0 . 0 4}$ & 23.25 & 21.40 & 17.59 & 25.14 & 23.40 & 24.94 \\
\hline $\mathbf{0 . 0 5}$ & 22.50 & 20.54 & 16.78 & 24.64 & 22.63 & 24.28 \\
\hline
\end{tabular}

\begin{tabular}{|l|l|l|l|l|l|l|}
\hline \multicolumn{7}{|c|}{ Table 3. Speckle Noise Filtering } \\
\hline $\begin{array}{l}\text { Speckle } \\
\text { Noise }\end{array}$ & $\begin{array}{l}\text { Average } \\
\text { Filter }\end{array}$ & $\begin{array}{l}\text { Median } \\
\text { Filter }\end{array}$ & $\begin{array}{l}\text { Adaptive } \\
\text { Median } \\
\text { Filter }\end{array}$ & $\begin{array}{l}\text { Arithmetic } \\
\text { Mean filter }\end{array}$ & $\begin{array}{l}\text { Contra } \\
\text { harmonic } \\
\text { Mean Filter }\end{array}$ & $\begin{array}{l}\text { Weiner } \\
\text { Filter }\end{array}$ \\
\hline $\begin{array}{l}\text { (In } \\
\text { percentage) }\end{array}$ & $P S N R$ & $P S N R$ & PSNR & PSNR & PSNR & PSNR \\
\hline $\mathbf{1 0 \%}$ & 24.54 & 21.19 & 18.12 & 25.82 & 24.78 & 24.09 \\
\hline $\mathbf{2 0} \%$ & 21.87 & 18.41 & 15.45 & 23.87 & 21.98 & 21.70 \\
\hline $\mathbf{3 0} \%$ & 20.32 & 16.83 & 13.95 & 22.57 & 20.38 & 20.39 \\
\hline $\mathbf{4 0} \%$ & 19.11 & 15.65 & 12.99 & 21.38 & 19.16 & 19.39 \\
\hline $\mathbf{5 0} \%$ & 18.38 & 14.80 & 12.57 & 20.60 & 18.42 & 18.85 \\
\hline
\end{tabular}



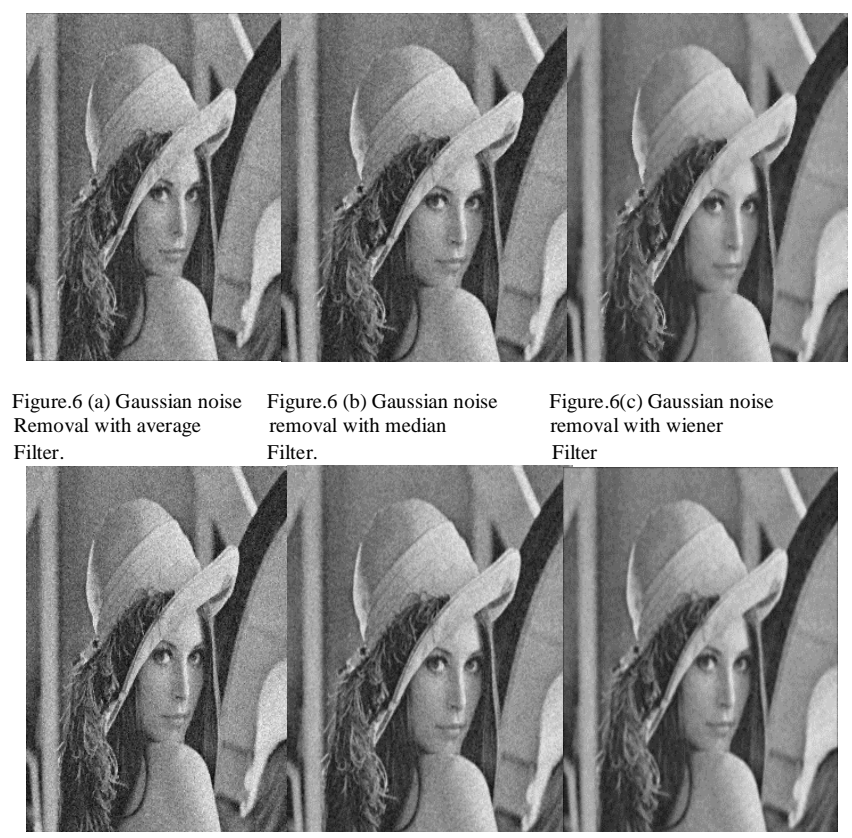
$\begin{array}{lll}\text { Figure.6 (d) Gaussian noise } & \text { Figure.6 (e) Gaussian noise } & \text { Figure.6 (f) Gaussian noise } \\ \text { Removal with adaptive } & \text { Removal with Contra } & \text { Removal with arithmetic } \\ \text { Median Filter. } & \text { Harmonic Filter. } & \text { Filter. }\end{array}$ Median Filter.

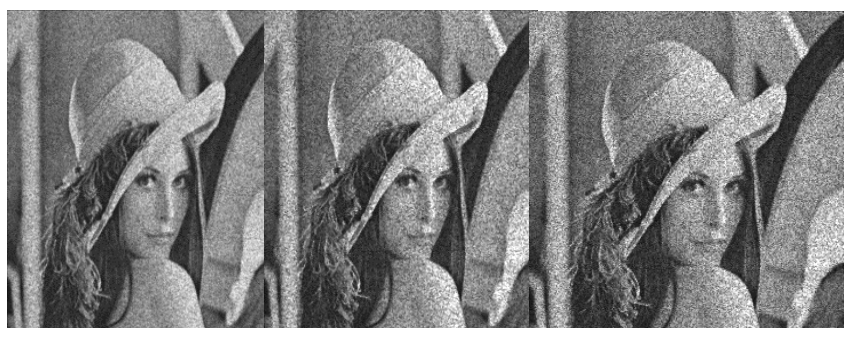

Figure.7 (a) Speckle noise Removal with mean Filter

Figure.7 (b) Speckle noise Removal with median Filter.

Figure.7(c) Speckle noise Removal with adaptive Median Filter

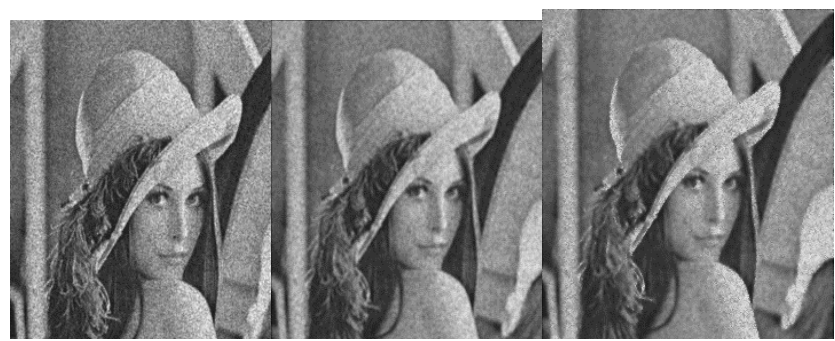

Figure.7 (d) Speckle noise Figure.7 (e) Speckle noise Removal with

Contra Harmonic Filter Mean Filter

Figure.7 (f) Speckle Noise Removal with Weiner Filter.

\section{CONCLUSION}

In this paper, different noise models their reduction techniques have been discussed. The six different type of filters which includes mean filter, median filter, adaptive median filter, contra harmonic mean filter, arithmetic mean filter and wiener filter were applied on three different type of noises which include salt and pepper noise, Gaussian noise and speckle noise. The performance of these denoising techniques is evaluated using reference based quality measures: PSNR and MSE. For analysis purposes, different range of noise densities are taken from $1 \%$ to $5 \%$. From Table 1, we can conclude that by increasing the noise density, PSNR value decreases. Considering the noise density 20\% in Table 1, we can see that adaptive median filter works very well in removing Salt and Pepper noise. From Table 2, we can notice that for Gaussian noise with variance 0.01 , Weiner filters performs well with high PSNR as compared to other filters. Similarly, in Table 3 arithmetic mean filter shows better performance in eliminating speckle noise.

\section{REFERENCES}

[1] Ruchika Shukla1 and Sugandha Agarwal2. Implementation of various image processing techniques, International Journal of Advance Computer Science and Technology (IJACST), Vol.5, May 2016.

[2] P.Patidar, and A.K.Nagawat. Image denoising by various filters for different noise, International Journal of Computer Applications, Vol. 9, November 2010.

[3] S. Suhaila, S. Z. H. A. Fakkri, R. Hazli, Z. Tukiran. Development of denoising method for digital image in low light condition, IEEE International Conference on Control System, Computing and Engineering, pp. 142-147, 2013.

[4] Lingli Huang. Improved nonlocal means algorithm for image denoising, Journal of Computer and Communications, Vol. 3, pp. 23-29, 2015.

https://doi.org/10.4236/jcc.2015.34003

[5] I. M. El-Henawy1, A. E. Amin2, Kareem Ahmed3, Hadeer Adel4. A comparative study on Image deblurring techniques, International Journal of Advances in Computer science and Technology (IJACST), Vol. 3, pp. 01-08, December 2014

[6] A. Jaiswal, J.Uphadhahy. Image denoising and quality measurements by using filtering and wavelet based techniques, International Journal of Electronics and Communications, Vol. 68, pp. 699-705, August 2014.

https://doi.org/10.1016/j.aeue.2014.02.003

[7] C. Saxena, D.Kaurov. Noises and image denoising techniques: A Brief Survey, International Journal of Emerging Technology and Advanced Engineering, Vol. 4, pp. 878-885, March 2014. 
[8] G.L.Wise, N. Gallagher. A Theoretical analysis of the properties of median filters, IEEE Transactions on Acoustics, Speech, and Signal Processing, Vol. 29, December 1981.

[9] A.Rachakonda, N.Sirinivas. Advanced technique for removal of salt \&amp; Pepper Noise in

Images, International Journal of Engineering and Computer Science, Vol. 2, pp. 2856-2860, September 2013.

[10] G.Ghimpe „T.Batard. A Decomposition Framework for Image Denoising Algorithms, IEEE Transactions On Image Processing, Vol. 25, January 2016.

[11] G.A. Eninike. Iterative filtering and smoothing of measurements possessing poison noise, IEEE transactions

On Aerospace and Electronic Systems, Vol. 51, pp. 22052211, 2015.

https://doi.org/10.1109/TAES.2015.140843

[12] Lena image, available at

https://www.cosy.sbg.ac.at/ pmeerw/Watermarking/lena.html.

[13] S.Zhong. Image denoising using wavelet thresholding and model selection, Image Processing, 2000 International Conference, Vol. 3, pp. 262, September 2000.

[14]A.Kumar, K.Joshi. A Review Paper: Noise models in digital image processing, Signal and Image Processing: An International Journal (SIPIJ), Vol. 6, April 2015.

[15]P.Kamboj, V.Rani. A brief study of various noise model and filtering techniques, Journal of Global Research in Computer Science, Vol. 4, April 2013.

[16] Q.Jhong, C.Lia, A method for wide density salt and pepper noise removal, Proc IEEE Control and decision conference, pp. 2940-2943, 2014.

[17] Y.Dong, R. H. Chan, and Shufang Xu. A detection statistic for random valued impulse noise, IEEE transaction on image processing, Vol. 16, April 2007.

https://doi.org/10.1109/TIP.2006.891348 